\title{
Atitudes perante os Trabalhadores mais Velhos: A Perspetiva dos Estudantes Universitários
}

\author{
Andreia Vitória ${ }^{1}$ \\ Arménio Rego \\ Católica Porto Business School \\ Universidade Católica Portuguesa \\ Madalena Vilas Boas \\ Universidade de Aveiro
}

\begin{abstract}
RESUMO - Procuramos compreender (a) se a estrutura pentadimensional (dimensões: adaptabilidade; valia dos mais velhos para a organização; conscienciosidade e lealdade; capital social e generosidade; desempenho) do instrumento de medida das atitudes dos gestores perante os trabalhadores mais velhos anteriormente desenvolvido é replicada em uma amostra de estudantes universitários e (b) se essas atitudes ajudam a explicar as decisões dos estudantes. A amostra envolveu 278 estudantes universitários portugueses. Os principais resultados são os seguintes: (a) a estrutura pentadimensional obtida com gestores portugueses e brasileiros replica-se na amostra de estudantes; (b) apesar de os estudantes reconhecerem qualidades nos trabalhadores mais velhos, revelam inclinação para práticas discriminatórias relativamente a esses trabalhadores, e essa inclinação parece ser mais acentuada do que a identificada em gestores.
\end{abstract}

Palavras-chave: trabalhadores seniores, discriminação etária, envelhecimento, relações entre gerações

\section{Attitudes towards Older Workers: The Perspective of University Students}

\begin{abstract}
We seek to understand (a) if the penta-dimensional structure (dimensions: adaptability, value of older people to the organization, conscientiousness and loyalty; capital and generosity; performance) of the measuring instrument of managers' attitudes towards older workers previously developed is replicated in a sample of university students and (b) whether these attitudes help to explain the decisions of students. The sample involved 278 university students. The main results are the following: (a) the penta-dimensional structure obtained with Portuguese and Brazilian managers is replicated in the sample of students; (b) although students recognize qualities in older workers, they tend to reveal discriminatory practices with respect to such employees, and those practices appear to be more pronounced than those observed with managers.
\end{abstract}

Keywords: older workers; age discrimination; aging, intergenerational relations

Ao longo das últimas décadas, têm-se observado alterações demográficas significativas, nomeadamente o decréscimo acentuado das taxas de natalidade e fecundidade. Tais alterações têm vindo a contribuir para o envelhecimento da população, o que tem impactos relevantes na vida e na gestão das organizações (e vice-versa, embora esse tema não seja aqui considerado). De fato, a esperança média de vida tem vindo a aumentar significativamente nos últimos anos (Phillips \& Siu, 2012; Skirbekk, Loichinger, \& Barakat, 2012). As pessoas vivem mais tempo e com mais saúde, preservando diversas capacidades de trabalho por um período de tempo mais longo (mesmo após a aposentadoria). Ao mesmo tempo, as taxas de natalidade e fecundidade declinaram fortemente (Comissão Europeia, 2011; Phillips \& Siu, 2012; Skirbekk et al., 2012). O peso dos jovens adultos na pirâmide etária tem decrescido continuamente, enquanto o número de idosos tem aumentado de forma a se esperar um crescimento superior a $15 \%$, até 2030 , na União Europeia (Schalk et al., 2010).

Inevitavelmente, a força de trabalho tem vindo a envelhecer, mas muitas organizações não gerem de forma

1 Endereço para correspondência: DEGEIT, Universidade de Aveiro, Campus Universitário de Santiago, Aveiro, Portugal. CEP: 3810-193 E-mail: atvitoria@ua.pt profícua os trabalhadores mais velhos, desperdiçando muitas das suas capacidades (Kanfer \& Ackerman, 2004; von Bonsdorff, 2011). Entre as principais razões desse "subaproveitamento", estão os estereótipos negativos e as crenças errôneas sobre as capacidades e motivações dos mais velhos. Esses preconceitos podem desencadear desentendimentos, falhas na comunicação e problemas de cooperação intergeracional. Disso podem resultar efeitos "autoconfirmatórios" (Schalk et al., 2010): como existem preconceitos relativamente aos mais velhos, estes desenvolvem menores sentidos de autovalor e de autoeficácia, e a consequência desses efeitos é a queda do desempenho; confirma-se, assim, a expectativa e geram-se espirais de efeitos negativos recorrentes.

A literatura (e.g., Cappelli \& Novelli, 2010; Leibold \& Voelpel, 2006; Posthuma \& Campion, 2009) sugere que os trabalhadores mais velhos apresentam várias qualidades positivas que, se negligenciadas ou subestimadas, poderão gerar efeitos perversos nos indivíduos, nas organizações e na sociedade. Com atitudes e práticas discriminatórias, as organizações podem estar a menosprezar oportunidades para melhorar o desempenho organizacional. A gestão, a motivação e a atração de trabalhadores mais velhos representam, hoje, desafios fundamentais que as organizações não podem deixar de enfrentar. As atitudes negativas dos 
gestores em relação a esses trabalhadores representam restrições significativas a esse desafio (Kadefors \& Hanse, 2012), pelo que merecem ser estudadas.

A investigação realizada por Rego, Vitória, Cunha, Tupinambá e Leal (2016) sugere que os gestores desenvolvem atitudes perante os trabalhadores mais velhos que podem ser interpretadas à luz de cinco dimensões: (a) "adaptabilidade", (b) "valia dos mais velhos para a organização", (c) "conscienciosidade e lealdade", (d) "capital social e generosidade" e (e) “desempenho". Esses autores também verificaram que tais atitudes explicam diversas decisões dos gestores relativamente à escolha de candidatos de diferentes idades em processos de seleção e formação. A evidência empírica obtida mostra ainda que, mesmo quando os candidatos mais velhos são descritos mais positivamente do que os mais novos, muitos gestores tendem a escolher/ preferir os mais jovens.

O presente estudo pretende investigar se essas tendências discriminatórias são, e em que medida, replicáveis em estudantes universitários. Sendo estes os futuros profissionais e gestores de maior relevo na vida social e na organizacional, importa compreender se estão ou não sujeitos aos mesmos tipos de enviesamentos antes mencionados, ou seja, qual a precocidade da representação. As principais questões de pesquisa são as seguintes: (a) É a estrutura dimensional das atitudes dos gestores perante os mais velhos replicável em estudantes do ensino superior? (b) Divergirão as atitudes dos estudantes das atitudes dos gestores? (c) Tomarão os estudantes decisões diferentes das dos gestores em cenários relacionados com a seleção/contratação, a participação em programas de formação e a continuidade na organização? (d) Serão essas decisões explicáveis pelas suas atitudes perante os mais velhos?

$\mathrm{O}$ artigo começa por expor uma breve revisão da literatura sobre as atitudes perante os trabalhadores mais velhos e suas possíveis consequências. Depois, explica o método, a que se seguem os resultados. A parte final do artigo discute as principais conclusões e aponta algumas implicações para a ação.

\section{Revisão da Literatura}

Os estereótipos negativos e as crenças erróneas sobre as capacidades e motivações dos trabalhadores mais velhos prevalecem em muitas organizações e na própria sociedade (Cuddy, Norton, \& Fiske, 2005; Nelson, 2005; Schalk et al., 2010). Esses preconceitos podem gerar efeitos perversos sobre as relações intergeracionais (Cappelli \& Novelli, 2010; Leibold \& Voelpel, 2006; Taylor \& Walker, 1998). Estereótipos negativos levam muitos indivíduos (principalmente os mais jovens) a considerar os trabalhadores mais velhos como (a) menos produtivos, (b) menos competentes, (c) menos comprometidos com o trabalho, (d) menos inovadores e menos dispostos a aceitar a introdução de novas tecnologias, (e) pouco interessados na aprendizagem e no desenvolvimento de novas competências, (f) cognitivamente menos capazes (e.g., Backes-Gellner, Schneider, \& Veen, 2011; Cappelli \& Novelli, 2010; Cuddy et al., 2005; Kanfer \& Ackerman, 2004; Kite, Stockdale,
Whitley, Jr., \& Johnson, 2005; Lyon \& Pollard, 1997; Maurer, Barbeite, Weiss \& Lippstreu, 2008) e (g) interessados, principalmente, em aposentar-se o mais cedo possível (Capowski, 1994; Gendron, 2011; Midtsundstad, 2001).

Consequentemente, muitas organizações tendem a (a) desinvestir na formação dos trabalhadores mais velhos (Villosio, 2008), (b) discriminar os mais velhos em processos de seleção (Daniel \& Heywood, 2007; Gringart, Helmes, \& Speelman, 2005; Posthuma, Wagstraff, \& Campion, 2012), e (c) transmitir a mensagem não-verbal de que os trabalhadores mais velhos "não têm valor" (Schalk et al., 2010). Essas práticas comprometem a autoeficácia dos mais velhos, reforçando as crenças e os estereótipos que sobre os mesmos recaem (Maurer et al., 2008; Nelson, 2005; Roberson \& Kulik, 2007), assim se desenvolvendo círculos viciosos organizacionais cujos efeitos perversos se reforçam mutuamente (Masuch, 1985).

O potencial assim desperdiçado pode ser significativo. A literatura (e.g., Backes-Gellner et al., 2011; Cappelli \& Novelli, 2010; Kanfer \& Ackerman, 2004; Schalk et al., 2010; Warr, 1994) sugere que os trabalhadores mais velhos (a) têm competências interpessoais e éticas elevadas, (b) possuem níveis de experiência, de conhecimento e de sabedoria com grande valia potencial para as equipes e as organizações, (c) evidenciam menor absenteísmo e maiores níveis de lealdade e compromisso para com a organização, (d) têm menos acidentes de trabalho e (e) não revelam menor desempenho no trabalho do que os mais jovens, podendo até evidenciar desempenho qualitativo superior. Há, pois, razões para supor que os trabalhadores mais velhos são, pelo menos, tão competentes quanto os mais jovens (Cuddy et al., 2005). Kadefors e Hanse (2012) argumentam que as fraquezas de alguns trabalhadores mais velhos (e.g., perda de funções cognitivas), potencialmente nefastas para o desempenho do seu trabalho, podem ser superadas por outras características positivas (e.g., menor absenteísmo, maior satisfação no trabalho, maior comprometimento).

A evidência empírica (e.g., Leibold \& Voelpel, 2006) também não confirma o estereótipo de que os trabalhadores mais velhos rejeitam a mudança. Tanto os mais jovens quanto os mais velhos podem resistir versus aderir às mudanças, dependendo essas reações não da idade, mas do modo como a mudança é conduzida. Ademais, e contrariamente ao que é frequentemente assumido (que os trabalhadores mais velhos evitam ou estão menos motivados para a formação), a maioria dos trabalhadores mais velhos aprecia continuar a aprender e a melhorar as suas capacidades (Capowski, 1994; Cappelli \& Novelli, 2010; Streb, Voelpel, \& Leibold, 2008). Em dissonância com essa realidade, a evidência empírica (Finkelstein, Burke, \& Raju, 1995; Leibold \& Voelpel, 2006; Posthuma et al., 2012) sugere que os trabalhadores mais velhos têm menos acesso à formação. Esse menor acesso resulta, em grande medida, da crença segundo a qual o investimento na formação dos mais velhos é menos rentável (Cappelli \& Novelli, 2010) porque o período de permanência dos mesmos na organização será menor, o que dificulta a rentabilização do investimento em formação.

Ao contrário do que é comummente presumido, muitos trabalhadores gostariam de continuar a trabalhar para além da idade da aposentadoria tradicional. Na verdade, 
progressivamente, terão mesmo de fazê-lo, a fim de sustentar a sua vida mais longa e saudável, de modo a manter o estilo de vida pré-aposentadoria (Billett, Dymock, Johnson, \& Martin, 2011; Midtsundstad, 2011; OECD, 2012).

O panorama descrito corrobora a visão de autores que consideram que uma força de trabalho envelhecida pode trazer benefícios para as organizações (Billett et al., 2011; DeLong, 2004; Kanfer \& Ackerman, 2004; Leibold \& Voelpel, 2006; Skirbekk et al., 2012; Streb et al., 2008; Tikkanen, 2011) e sugerem que as organizações podem perder oportunidades para melhorar o seu desempenho se não gerirem devidamente os trabalhadores mais velhos. $\mathrm{O}$ problema pode tornar-se ainda mais pronunciado devido aos desequilíbrios nos sistemas de previdência social, que têm vindo a pressionar o poder político a aumentar a idade da aposentadoria.

Em suma, as organizações, os indivíduos e a própria sociedade têm de lidar eficazmente com esta realidade emergente: o envelhecimento da força de trabalho. O modo como os gestores encaram os trabalhadores mais velhos pode ter um efeito significativo na maneira como lidam com essa realidade. Daí a pertinência de se estudarem as respetivas atitudes. O tópico está escassamente investigado (e.g., Cappelli \& Novelli, 2010; Kanfer \& Ackerman, 2004), embora investigação recente realizada por Rego et al. (2016), com gestores portugueses e brasileiros, seja promissora. Os autores desenvolveram e validaram um instrumento de medida das atitudes dos gestores perante os trabalhadores mais velhos, tendo sugerido uma estrutura dimensional contendo cinco tipos de atitudes: (a) "adaptabilidade", (b) "valia dos mais velhos para a organização", (c) "conscienciosidade e lealdade", (d) "capital social e generosidade" e (e) "desempenho". A atitude "adaptabilidade" representa o grau em que os gestores consideram os mais velhos como mais/menos adaptáveis e mais/menos capazes de aprender. A atitude "valia dos mais velhos" representa o grau em que os gestores consideram os conhecimentos e a experiência dos mais velhos como valiosos para as organizações. A atitude "conscienciosidade e lealdade" reflete a medida em que os gestores entendem os mais velhos como mais/menos conscienciosos e leais à organização. A atitude "capital social e generosidade" diz respeito ao modo como os gestores avaliam a capacidade dos mais velhos de desenvolverem capital social (ou seja, melhores relacionamentos interpessoais) e de darem mostras de generosidade na relação com os demais membros organizacionais. A atitude "desempenho" reflete o grau em que os gestores consideram os mais velhos como mais/menos produtivos e reveladores de maior/menor desempenho.

Essa estrutura dimensional (Rego et al., 2016) representa progressos relativamente a medidas anteriores. Por exemplo, Maurer et al. (2008) sugeriram apenas dois tipos de "crenças" sobre os mais velhos: (a) capacidade e (b) desejo de aprendizagem e desenvolvimento. Hassell e Perrewe (1995) propuseram um rol de "crenças" sobre os mais velhos, mas não procederam à análise dimensional. Chiu, Chan, Snape e Redman (2001) usaram uma parte dos itens propostos por Hassell e Perrewe (1995), tendo evidenciado uma estrutura bidimensional: (a) adaptabilidade e (b) eficácia no trabalho.
Ademais, investigaram atitudes dos empregados em geral, não de gestores.

Rego et al. (2016) verificaram que as cinco atitudes referidas explicam o modo como, em cenários hipotéticos, os gestores selecionam os trabalhadores mais velhos versus os mais jovens em processos de seleção e de formação. Notaram, ainda, que o padrão empírico evidenciado em uma amostra de gestores portugueses é replicado em uma amostra de gestores brasileiros. E mostraram que, apesar de reconhecerem qualidades positivas nos trabalhadores mais velhos, muitos gestores tomam decisões discriminatórias. Neste estudo, procuramos investigar se esses efeitos são igualmente identificáveis em estudantes universitários. Mais especificamente, testamos se (a) a estrutura pentadimensional do instrumento de medida validado com gestores é replicada em uma amostra de estudantes universitários e (b) se essas atitudes também ajudam a explicar as decisões dos estudantes em cada cenário apresentado (tal como se verificou com os gestores).

O tema é relevante porque esses estudantes virão a assumir funções de maior importância na sociedade e nas organizações. Conhecer as suas atitudes e as consequências das mesmas permite melhor compreender o que poderá suceder quando tais indivíduos assumirem funções organizacionais. A evidência assim obtida pode também ajudar a identificar possíveis ações de sensibilização no contexto universitário, nomeadamente no ensino da gestão. Naturalmente, o relacionamento entre mais jovens e mais velhos é um caminho de duplo sentido, pelo que o estudo das atitudes dos mais velhos relativamente aos mais jovens é igualmente pertinente - mas esse não é o foco do presente estudo.

\section{Método}

\section{Participantes e Procedimentos}

O estudo foi levado a cabo, entre setembro e outubro de 2013, com estudantes de primeiro e segundo ciclos (Universidade de Aveiro - Portugal) que frequentavam cursos de (a) gestão, (b) engenharia e gestão industrial, (c) línguas e relações empresariais, (d) novas tecnologias da comunicação e (e) turismo. Todos esses cursos envolvem componentes de gestão, e muitos estudantes acabam por, após o curso, exercer funções de gestão nas suas organizações. A coleta de dados foi realizada em sala de aula, após autorização dos docentes (e dos respetivos alunos). Foram observados os princípios consagrados no Código de Ética da APA (2002).

Participaram 278 estudantes (53\% do sexo feminino), de forma anônima (as respostas foram entregues em envelope fechado diretamente aos pesquisadores). As idades situavam-se entre os 18 e os 49 anos. Todavia, mais de $72 \%$ dos estudantes tinham entre 18 e 23 anos, pelo que a média etária é de 22,1 anos (desvio-padrão: 3,8). Os estudantes foram primeiramente convidados a responder ao questionário de medida das suas atitudes perante os trabalhadores mais velhos, após o que lhes foi solicitado que tomassem decisões em quatro cenários que envolviam a 
escolha entre trabalhadores mais velhos versus mais jovens. Nas duas seções seguintes, explanamos o conteúdo desses instrumentos de medida.

\section{Instrumento de Medida das Atitudes perante os Mais Velhos}

As atitudes dos alunos perante os trabalhadores mais velhos foram medidas através do questionário previamente desenvolvido e validado por Rego et al. (2016), contendo 20 itens e medindo as cinco dimensões antes referidas. Aos vinte itens foram adicionados seis - emergentes da investigação realizada por Rego et al. (2016), assim como da revisão da literatura -, tendo como objetivo melhorar as propriedades psicométricas do questionário. Desse modo, procuramos (a) incrementar os índices de consistência interna (Alphas de Cronbach) e (b) harmonizar a quantidade de itens medindo cada dimensão/fator, para reforçar a sua "força equivalente" (Cooper \& Richardson, 1986). Foi solicitado aos alunos que descrevessem o seu nível de concordância com as 26 afirmações contidas no questionário, mediante uma escala de sete pontos: 1 - "A afirmação é completamente falsa"; (...); 7 - "A afirmação é completamente verdadeira".

Foi levada a cabo uma análise fatorial confirmatória (LISREL; método de estimação da máxima verosimilhança), testando-se o modelo de cinco fatores antes referido. Os índices de ajustamento (Tabela 1, coluna "Modelo Inicial") não se revelaram satisfatórios (e.g., RMSEA: 0,08; GFI: 0,82; CFI: 0,75 e IFI: 0.76; Byrne, 1998). Alguns Lambdas e alguns Alfas de Cronbach também emergiram com valores inferiores ao recomendável (0,50 e 0,70, respetivamente). Após análise dos índices de modificação e dos resíduos estandardizados e tendo em atenção os valores dos Lambdas, foram removidos sete itens (ver os 19 itens remanescentes na coluna "Modelo Final", Tabela 1). Dos seis itens adicionados, apenas dois permanecem no modelo final. Dos anteriores vinte itens (Rego et al., 2016), foram excluídos três. Os índices de ajustamento do modelo contendo os 19 itens finais são razoavelmente satisfatórios (e.g., RMSEA: 0,06; GFI: 0,90; CFI: 0,88 e IFI: 0.88; Byrne, 1998). Os Lambdas são superiores a 0,5 , com exceção de um, cujo valor $(0,47)$ é muito próximo desse patamar. Os Alfas de Cronbach das dimensões adaptabilidade, desempenho, e capital social e lealdade são superiores a 0,70 e os das restantes dimensões (valia dos mais velhos; conscienciosidade e lealdade) encontram-se próximos desse valor, o que para alguns autores é considerado aceitável (Price \& Mueller, 1986).

\section{Variáveis Dependentes}

Para testar a capacidade preditiva das atitudes dos estudantes perante os trabalhadores mais velhos, foram criados quatro cenários: (a) um referente à seleção/ contratação, (b) outro relacionado com a seleção de trabalhadores para participar em formação, (c) outro envolvendo uma hipotética contratação de um jovem recémformado versus a continuidade do trabalhador atual, mais velho, após a sua aposentadoria, e (d) o último relativo à escolha do trabalhador jovem versus o mais velho para abandonar a organização num processo de downsizing. Os três primeiros cenários foram usados por Rego et al. (2016).

O teor do primeiro cenário foi o seguinte: "Duas pessoas, com iguais qualificações, candidatam-se a um cargo na sua organização. A primeira (A) tem 26 anos, a segunda (B) tem 51. Quem escolheria?”. Enquanto nesse cenário só a idade diferia na descrição de ambos os trabalhadores, nos cenários seguintes foram incluídas características que favoreciam o perfil do trabalhador mais velho. A literatura (Kite et al., 2005) sugere que o preconceito relativo à idade é enfraquecido quando é fornecida informação adicional (além da idade) acerca do trabalhador. O conteúdo do segundo cenário era o seguinte: "A sua empresa tem que escolher, entre dois colaboradores (A e B), qual participará de uma formação que uma universidade oferece gratuitamente. Ambos têm bom desempenho e as mesmas habilitações. Todavia, o colaborador A, mais motivado para essa formação, tem 51 anos, enquanto o colaborador B tem apenas 26. Quem escolheria?". O terceiro cenário foi redigido da seguinte forma: "Uma pessoa jovem recém-licenciada candidatase a uma função técnica (área financeira). A pessoa que agora exerce a função, também licenciada, está prestes a reformar-se, mas mostra interesse em continuar a exercê-la em regime de prestação de serviços, com uma compensação inferior à que seria paga à recém-licenciada. Contrataria a pessoa recém-licenciada?". Por fim, o teor do quarto cenário era o seguinte: "Numa empresa do setor alimentar, duas pessoas, com as mesmas habilitações, exercem funções administrativas similares. Uma (A) tem 56 anos e apresenta um elevado desempenho. A outra (B) tem 25 anos e apresenta desempenho menor. Para reduzir custos, só uma poderá continuar. Qual delas escolheria para sair?"

Importa esclarecer as duas razões pelas quais foram atribuídas idades distintas aos trabalhadores descritos nos cenários. Primeira: pretendia-se criar verosimilhança dos cenários, no seu conjunto. Ou seja, o eventual uso das mesmas idades nos quatro cenários poderia reforçar a sensação de artificialidade na mente dos respondentes. Segunda: a literatura não é consistente na definição de "trabalhador mais velho", não estando claramente definida a idade a partir da qual um trabalhador passa a ser considerado como "mais velho" (Finkelstein, Ryan, \& King, 2013). Por exemplo, Maurer et al. (2008) consideram os trabalhadores mais velhos como aqueles cuja idade excede os 40 anos. No entanto, outros autores usaram os 35 (Ashbaugh \& Fay, 1987), os 50 (Finkelstein et al., 2013; OCDE, 2006) ou os 55 anos (Ekamper, 2010) como limite.

\section{Análise de dados}

Além da já mencionada análise fatorial confirmatória (efetuada através do LISREL, versão 9.2), foram realizadas diversas outras análises estatísticas (mediante o SPSS 21): análise de correlações, análise de variância, e análise de regressão logística binária (dada a natureza binária das variáveis dependentes). 


\section{Resultados}

\section{Médias, Desvios-Padrão e Correlações}

A Tabela 2 evidencia as médias, os desvios-padrão e as correlações. Atendendo à amplitude da escala usada (1-7) para medir as atitudes dos gestores perante os trabalhadores mais velhos, pode-se afirmar que as atitudes sobre "conscienciosidade e lealdade" e sobre o "desempenho" são moderadamente positivas, uma vez que a pontuação média é superior a 5 . A atitude relativa à "valia" dos trabalhadores mais velhos pode ser considerada moderada e as demais atitudes podem ser consideradas moderadamente baixas. A idade dos estudantes não se correlaciona significativamente com nenhuma das atitudes,

Tabela 1. Análise fatorial confirmatória

\begin{tabular}{|c|c|c|c|c|c|}
\hline \multirow[t]{2}{*}{ Dimensão } & \multirow[t]{2}{*}{ Item } & \multicolumn{2}{|c|}{$\begin{array}{l}\text { Modelo } \\
\text { Inicial }\end{array}$} & \multicolumn{2}{|c|}{$\begin{array}{c}\text { Modelo Final } \\
\text { (após remoção } 7 \\
\text { itens) }\end{array}$} \\
\hline & & $\beta$ & $\alpha$ & $\beta$ & $\alpha$ \\
\hline \multirow[t]{6}{*}{ Adaptabilidade } & Os mais velhos adaptam-se melhor às mudanças organizacionais.* & 0,07 & & - & \\
\hline & Os trabalhadores mais velhos têm menos capacidade de adaptação a novas situações. & $-0,59$ & & 0,55 & \\
\hline & $\begin{array}{l}\text { Os mais velhos demoram mais tempo a tornar-se competentes no exercício de uma nova } \\
\text { função. }\end{array}$ & $-0,73$ & & 0,74 & \\
\hline & Os trabalhadores mais velhos resistem mais à mudança. & $-0,41$ & 0,68 & - & 0,70 \\
\hline & Os mais velhos são menos criativos/inovadores. & $-0,61$ & & 0,62 & \\
\hline & $\begin{array}{l}\text { Os trabalhadores mais velhos têm menos vontade de participar em programas de } \\
\text { formação. }\end{array}$ & $-0,55$ & & 0,53 & \\
\hline \multirow[t]{5}{*}{ Valia dos mais velhos } & A experiência dos trabalhadores mais velhos é pouco valiosa para as organizações atuais. & 0,17 & & - & \\
\hline & Os mais velhos raciocinam mais lentamente. & 0,59 & & 0,59 & \\
\hline & $\begin{array}{l}\text { As competências dos mais velhos são mais importantes para o sucesso das organizações } \\
\text { do que as dos mais novos.* }\end{array}$ & 0,50 & 056 & 0,47 & 60 \\
\hline & $\begin{array}{l}\text { Os mais velhos são menos valiosos para as empresas pois são menos competentes em } \\
\text { novas tecnologias. }\end{array}$ & 0,56 & & 0,57 & \\
\hline & $\begin{array}{l}\text { As características dos mais velhos são menos valiosas para as organizações do que as } \\
\text { características dos mais jovens. }\end{array}$ & 0,54 & & 0,56 & \\
\hline \multirow{5}{*}{$\begin{array}{l}\text { Conscienciosidade e } \\
\text { lealdade }\end{array}$} & Os mais velhos faltam mais ao trabalho. & 0,24 & & - & \\
\hline & Os mais velhos importam-se com o futuro das suas organizações.* & 0,41 & & - & \\
\hline & Os mais velhos sentem menos vontade de assumir responsabilidades no trabalho. & 0,62 & 049 & 0,59 & 060 \\
\hline & $\begin{array}{l}\text { Os trabalhadores mais velhos estão menos dispostos a fazer sacrifícios em nome da } \\
\text { organização. }\end{array}$ & 0,72 & & 0,57 & \\
\hline & Os mais velhos são menos leais à organização. & 0,67 & & 0,54 & \\
\hline \multirow{5}{*}{$\begin{array}{l}\text { Capital social e } \\
\text { generosidade }\end{array}$} & Os mais velhos estão mais dispostos a ajudar os colegas de trabalho. & 0,37 & & 0,63 & \\
\hline & $\begin{array}{l}\text { Os mais velhos são capazes de estabelecer relacionamentos mais duradouros com outras } \\
\text { pessoas. }\end{array}$ & 0,19 & & 0,51 & \\
\hline & Em geral, os mais velhos são mais cooperantes. & 0,59 & 073 & 0,67 & 073 \\
\hline & $\begin{array}{l}\text { Os trabalhadores mais velhos gostam mais de sentir que o seu trabalho é útil para a } \\
\text { sociedade. }\end{array}$ & 0,52 & & 0,54 & \\
\hline & $\begin{array}{l}\text { Os mais velhos são mais generosos (por exemplo, preocupam-se mais com o bem-estar } \\
\text { dos outros) do que os mais jovens.* }\end{array}$ & 0,48 & & 0,65 & \\
\hline \multirow[t]{5}{*}{ Desempenho } & Os mais velhos são mais competentes e eficazes na realização do seu trabalho.* & 0,63 & & - & \\
\hline & Os mais velhos são mais competentes na realização das suas tarefas.* & 0,51 & & - & \\
\hline & As pessoas mais velhas fazem trabalho com pior qualidade. & 0,66 & 0,66 & 0,61 & 0,71 \\
\hline & Em geral, os mais velhos têm pior desempenho. & 0,54 & & 0,74 & \\
\hline & Os trabalhadores mais velhos são menos produtivos. & 0,66 & & 0,70 & \\
\hline \multicolumn{2}{|l|}{ Qui-Quadrado } & \multicolumn{2}{|c|}{784,13} & \multicolumn{2}{|c|}{298,64} \\
\hline \multicolumn{2}{|l|}{ Graus de Liberdade } & \multicolumn{2}{|c|}{289} & \multicolumn{2}{|c|}{142} \\
\hline \multicolumn{2}{|c|}{ Qui-Quadrado/Graus de Liberdade } & \multicolumn{2}{|c|}{2,71} & \multicolumn{2}{|c|}{2,10} \\
\hline \multicolumn{2}{|l|}{ RMSEA } & \multicolumn{2}{|c|}{0,08} & \multicolumn{2}{|c|}{0,06} \\
\hline \multicolumn{2}{|c|}{ Goodness of Fit Index (GFI) } & \multicolumn{2}{|c|}{0,82} & \multicolumn{2}{|c|}{0,90} \\
\hline \multicolumn{2}{|c|}{ Comparative Fit Index (CFI) } & \multicolumn{2}{|c|}{0,75} & \multicolumn{2}{|c|}{0,88} \\
\hline \multicolumn{2}{|c|}{ Incremental Fit Index (IFI) } & \multicolumn{2}{|c|}{0,76} & & \\
\hline
\end{tabular}

Nota: * Item novo 
nem com qualquer decisão contemplada nos cenários. O sexo correlaciona-se negativamente com as atitudes "adaptabilidade" e "desempenho", bem como com a decisão relativa ao primeiro e ao terceiro cenários: (a) os homens tendem a descrever um trabalhador mais velho de forma menos positiva no que diz respeito àquelas duas dimensões, (b) são menos propensos a contratá-los, no primeiro cenário e (c) tendem a escolher o recém-formado (em detrimento do trabalhador mais velho) no terceiro cenário.

As cinco dimensões representando as atitudes perante os trabalhadores mais velhos interrelacionam-se significativamente, apenas se excetuando a correlação entre a "valia dos mais velhos" e o "capital social e generosidade". A "adaptabilidade" correlaciona-se positivamente com a "valia dos mais velhos", com a "conscienciosidade e lealdade" e com o "capital social e generosidade", mas negativamente com o "desempenho". Por conseguinte, os estudantes que consideram os trabalhadores mais velhos como mais adaptáveis tendem a valorizar mais as respetivas competências e a considerá-los como mais conscienciosos e leais, mas atribuem-lhes pior desempenho. A "valia dos mais velhos" correlaciona-se positivamente com a "conscienciosidade e lealdade" e com o "desempenho". A atitude "conscienciosidade e lealdade" correlaciona-se positivamente com o "desempenho", mas negativamente com o "capital social e generosidade". Na perspetiva dos estudantes, os trabalhadores seniores mais conscienciosos e leais revelam melhor desempenho, mas atribuem-lhes níveis inferiores de "capital social e generosidade". O "capital social e generosidade" correlaciona-se negativamente com o "desempenho". Ou seja, os estudantes que consideram os trabalhadores mais velhos como mais generosos e "relacionais" tendem a atribuir-lhes pior desempenho.

Três das cinco dimensões (valia dos mais velhos; capital social e generosidade; desempenho) correlacionam- se positivamente com a preferência dos estudantes pelo candidato mais velho em um cenário de contratação/ seleção (primeiro cenário), enquanto o sexo e a atitude "adaptabilidade" se correlacionam negativamente com a decisão. A atitude sobre o "desempenho" correlacionase de forma positiva com a opção dos estudantes pelo trabalhador mais velho para ingressar em um programa de formação (segundo cenário). No que diz respeito à decisão do terceiro cenário (contratação do recém-formado versus continuidade do mais velho depois da aposentadoria), as mulheres apresentam maior propensão para contratarem o trabalhador/candidato mais velho. A atitude "adaptabilidade" correlaciona-se positivamente com a decisão dos alunos de escolherem o trabalhador mais velho para sair da organização em um cenário de downsizing (quarto cenário).

\section{As Atitudes Predizendo as Decisões Contempladas nos Cenários}

A Tabela 3 apresenta a percentagem de estudantes que escolheu o trabalhador mais velho versus o mais novo em cada cenário, bem como as correspondentes pontuações médias em cada atitude. No primeiro cenário, 80\% dos estudantes escolheu contratar o trabalhador mais jovem. Os $20 \%$ de estudantes que optaram pelo mais velho apresentam pontuações médias mais elevadas nas cinco atitudes estudadas. No segundo cenário, 73\% dos estudantes selecionaram o trabalhador mais velho para participar no programa de formação, enquanto $27 \%$ optaram pelo mais novo, mas menos motivado. As atitudes dos alunos que escolheram o trabalhador mais velho são muito similares às dos estudantes que escolheram o mais jovem, sendo apenas ligeira e significativamente superiores na atitude "desempenho". No terceiro cenário, 57\% dos estudantes

Tabela 2. Médias, desvios-padrão e correlações

\begin{tabular}{|c|c|c|c|c|c|c|c|c|c|c|c|c|}
\hline & $\mathbf{M}$ & DP & 1 & 2 & 3 & 4 & 5 & 6 & 7 & 8 & 9 & 10 \\
\hline 1.Idade & 22,05 & 3,84 & - & & & & & & & & & \\
\hline 2. Género (a) & & & $0,16^{*}$ & - & & & & & & & & \\
\hline 3. Adaptabilidade & 3,92 & 1,05 & $-0,02$ & $-0,22^{* * *}$ & - & & & & & & & \\
\hline 4. Valia dos mais velhos & 4,48 & 0,70 & $-0,02$ & $-0,10$ & $0,36^{* * *}$ & - & & & & & & \\
\hline 5. Conscienciosidade e lealdade & 5,31 & 0,91 & $-0,11$ & $-0,12$ & $0,33 * * *$ & $0,28 * * *$ & - & & & & & \\
\hline 6. Capital social e generosidade & 4,05 & 0,89 & $-0,03$ & 0,08 & $0,36^{* * *}$ & $-0,12$ & $-0,14^{*}$ & - & & & & \\
\hline 7. Desempenho & 5,46 & 0,93 & $-0,04$ & $-0,15^{*}$ & $0,46^{* * *}$ & $0,46^{* * *}$ & $0,54^{* * *}$ & $-0,21 * *$ & - & & & \\
\hline $\begin{array}{l}\text { 8. Cenário \# 1: Contratar o trabalhador mais velho } \\
\text { versus o mais jovem. (b) }\end{array}$ & - & - & 0,00 & $-0,14^{*}$ & $0,17 * *$ & $0,17 * *$ & 0,12 & $0,15^{*}$ & $0,14^{*}$ & - & & \\
\hline $\begin{array}{l}\text { 9. Cenário \# 2: Selecionar o trabalhador mais } \\
\text { velho versus o mais jovem, para um programa de } \\
\text { formação. (b) }\end{array}$ & - & - & $-0,06$ & $-0,21$ & 0,04 & 0,06 & 0,10 & $-0,06$ & $0,15^{*}$ & $-0,04$ & - & \\
\hline $\begin{array}{l}\text { 10. Cenário \# 3: Contratar o trabalhador mais } \\
\text { velho (depois de se aposentar) versus o mais } \\
\text { jovem (b) }\end{array}$ & - & - & $-0,08$ & $-0,13^{*}$ & $-0,06$ & 0,02 & 0,02 & 0,10 & $-0,01$ & $-0,03$ & $-0,08$ & - \\
\hline $\begin{array}{l}\text { 11. Cenário \# 4: Selecionar o trabalhador } \\
\text { mais velho versus o mais jovem para sair da } \\
\text { organização num processo de downsizing. (b) }\end{array}$ & - & - & $-0,06$ & $-0,02$ & $0,13^{*}$ & $-0,05$ & 0,05 & $-0,05$ & 0,05 & 0,03 & $-0,11$ & 0,06 \\
\hline
\end{tabular}


escolheram contratar um trabalhador mais jovem, a quem seria pago um salário superior, em detrimento de manter o trabalhador mais velho, depois de este se aposentar. Não se detectaram diferenças estatisticamente significativas entre as atitudes dos dois grupos de estudantes. No quarto cenário, $60 \%$ dos estudantes selecionaram o trabalhador mais jovem para sair da organização em um processo de downsizing. Esses estudantes evidenciam uma atitude mais favorável relativamente aos mais velhos no que concerne à dimensão "adaptabilidade".

Foi levada a cabo uma regressão logística binária para testar o valor preditivo das atitudes para as decisões dos estudantes perante cada cenário. A tabela 4 mostra que as atitudes dos estudantes em relação aos trabalhadores mais velhos predizem 10\% (R2 de Cox \& Snell) ou 15\% (R2 de Nagelkerke) da variância da decisão tomada no primeiro cenário. Os fatores preditores da decisão no primeiro cenário são as atitudes "adaptabilidade" e "capital social e generosidade". As atitudes dos estudantes explicam, igualmente, variância (embora reduzida) da decisão no segundo cenário $(4 \%, \mathrm{R} 2$ de Cox \& Snell; 5\%, R2 de Nagelkerke). Para o segundo cenário, o único fator preditivo significativo é a atitude "desempenho". No que respeita ao terceiro cenário, as atitudes dos alunos explicam, de forma modesta, a variância das suas decisões $(2 \%$, R2 de Cox \& Snell; 3\%, R2 de Nagelkerke), nenhuma atitude apresentando

Tabela 3. Comparação entre os estudantes que selecionaram o trabalhador mais velho versus os que selecionaram o trabalhador mais $\underline{\text { novo }}$

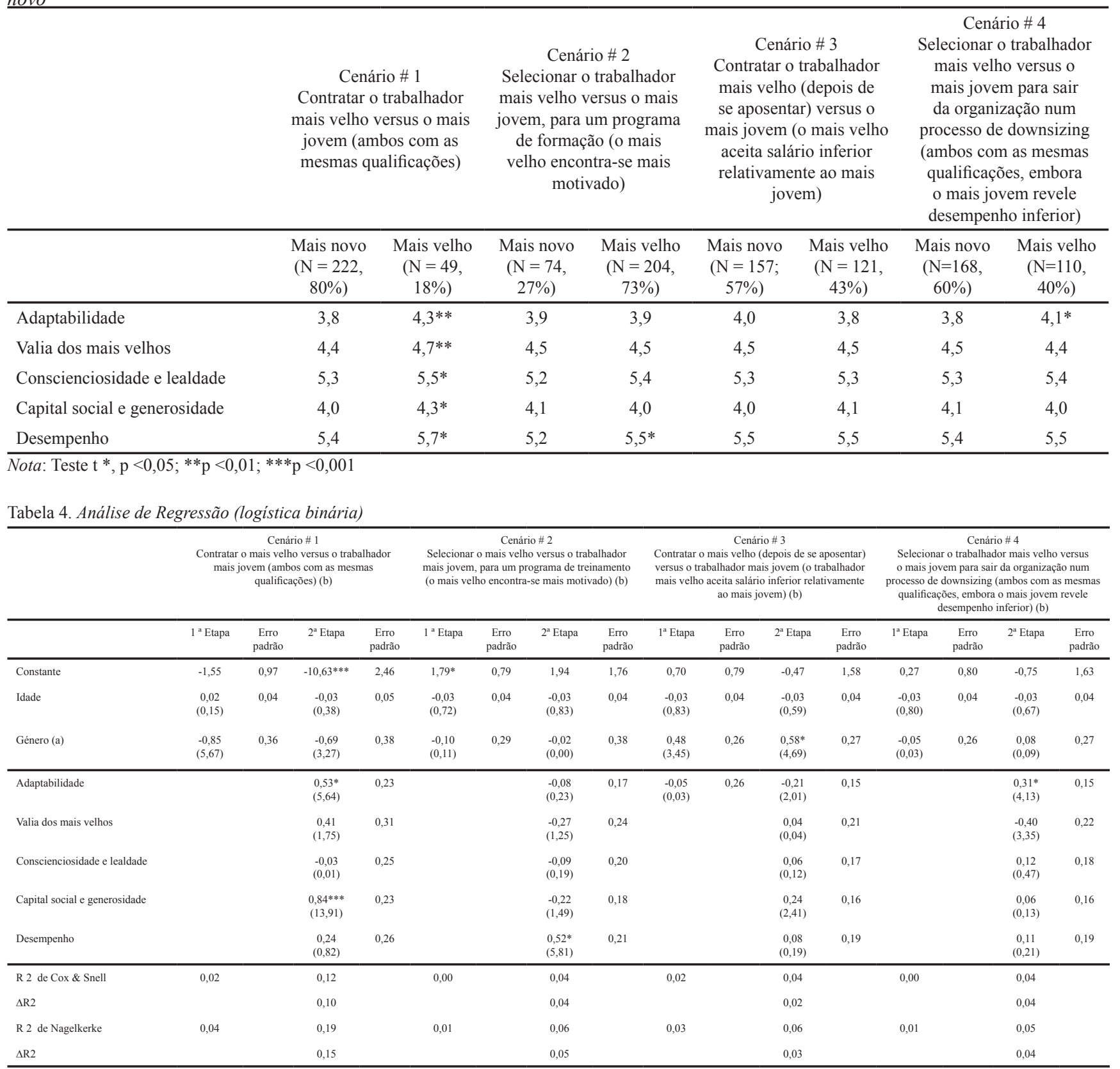

Notas. $\mathrm{N}=278 ;{ }^{*} p<0,05 ;{ }^{* *} p<0,01 ; * * * p<0,001$; Valor entre parênteses: estatística Wald; (a) Sexo: feminino ( 0 ); masculino (1); (b) Escolher o trabalhador mais velho (1) versus escolher o mais novo (0) 
poder preditivo significativo. Todavia, o sexo dos inquiridos prediz a decisão relativa a esse cenário. Por fim, no quarto cenário, as atitudes dos estudantes explicam 4\% (R2 de Cox \& Snell, e R2 de Nagelkerke) da variância da decisão, sendo a "adaptabilidade" o único fator preditivo.

\section{Análise, Discussão e Conclusões}

\section{Formando Sentido dos Principais Resultados}

A análise dos resultados permite identificar cinco principais aspetos. Em primeiro lugar, a análise fatorial confirmatória sugere que o modelo de cinco atitudes/fatores se ajusta aos dados de forma satisfatória. Essa evidência corrobora os resultados dos estudos realizados por Rego et al. (2016) com amostras de gestores em dois países distintos e sugere que o modelo pentadimensional é porventura transversal a várias populações. Importa notar, todavia, que o Alfa de Cronbach de duas dimensões ("conscienciosidade e lealdade": 0,60; "valia dos mais velhos": 0,63) é menor do que o valor de corte de 0,70. Acrescente-se que (a) dos seis itens adicionados ao instrumento original (Rego et al., 2016), apenas dois permaneceram no modelo final e (b) dos vinte itens originais, excluíram-se três. Por conseguinte, a estrutura de cinco fatores parece representar os dados de forma adequada e, globalmente, estudantes e gestores percebem os mais velhos a partir de perspetivas condizentes. Todavia, é também possível que cada população use (alguns) indicadores distintos quando avaliam as qualidades dos trabalhadores seniores. Por conseguinte, estudos futuros deverão testar a inserção de novos itens de modo a (a) melhorar a consistência interna de algumas escalas, (b) garantir igual representatividade a todas as dimensões (Cooper \& Richardson, 1986), e (c) identificar escalas de aplicação transversal a diversos tipos de respondentes.

Em segundo lugar, os dados sugerem que as atitudes dos estudantes, comparativamente com as dos gestores inquiridos por Rego et al. (2016), são similares (adaptabilidade; conscienciosidade; desempenho) ou menos positivas (valia dos mais velhos; capital social e generosidade). Os dados corroboram, parcialmente, os de Rego et al. (2016), nos quais foi identificada correlação positiva entre a idade dos respondentes e diversas atitudes perante os trabalhadores mais velhos (sobretudo na amostra de gestores portugueses). Essa evidência também é consistente com a literatura (e.g., Hassell \& Perrewe, 1995; Munnel, Sass, \& Soto, 2006), sugerindo que os indivíduos com idade superior tendem a ter opiniões mais favoráveis acerca dos trabalhadores mais velhos. Tal evidência pode ser explicada à luz dos efeitos de similitude (Larkey \& Markman, 2005; Liviatan, Trope, \& Liberman, 2008), ou com base na teoria de enviesamento em prol do endogrupo, que sugere que as pessoas mais velhas percebem os trabalhadores mais velhos de forma mais favorável, com o objetivo de perpetuar uma identidade social positiva como membros do grupo dos trabalhadores mais velhos (Chiu et al., 2001; Munnell et al., 2006). Note-se, todavia, que esse argumento se baseia na comparação entre os dados deste estudo e os de Rego et al. e não na comparação entre os estudantes de diferentes idades que participaram no nosso estudo. De fato, não encontramos aqui correlação significativa entre a idade e as atitudes (Tabela 2), sendo essa evidência porventura explicada pela reduzida variabilidade nas idades dos estudantes.

Em terceiro lugar, os dados não corroboram o estereótipo aludido por Cuddy et al. (2005), segundo o qual os trabalhadores mais velhos são "mais simpáticos mas incompetentes". De fato, e validando a evidência obtida por Rego et al. (2016; tabela 5), a cotação média na atitude "desempenho" é superior à pontuação média na atitude "capital social e generosidade". Todavia, a correlação negativa entre as duas atitudes (Tabela 2) sugere uma eventual tendência dos respondentes para considerarem que o incremento/decréscimo numa dessas qualidades está associado ao decréscimo/incremento na outra. Essa correlação negativa foi identificada nas duas amostras de gestores inquiridas por Rego et al., mas nelas emergiu um dado que, em certa medida, é consistente com o verificado na nossa amostra: a correlação negativa entre as atitudes "valia dos mais velhos para a organização" e "capital social e generosidade". Por conseguinte, nas duas amostras de Rego et al. (2016) e neste estudo, um incremento na atitude "desempenho" tem como corolário o decréscimo nas atitudes relacionadas com o desempenho ou a valia dos trabalhadores para a organização e vice-versa.

Em quarto lugar, comparativamente com os gestores inquiridos por Rego et al. (2016), os estudantes da nossa amostra tomam decisões perante dois cenários que são menos favoráveis para com os mais velhos. No cenário $1,80 \%$ dos estudantes escolheriam o trabalhador mais novo, sendo essa percentagem de $55 \%$ na amostra de gestores portugueses e de $56 \%$ na amostra de gestores brasileiros inquiridos por Rego et al.. No cenário 3, essas percentagens são, respetivamente, $57 \%, 46 \%$, e $34 \%$. No cenário 4 deste estudo, que não foi considerado por Rego et al., a percentagem de estudantes

Tabela 5. Atitudes perante os trabalhadores mais velhos

\begin{tabular}{cccc}
\hline & Amostra do presente estudo & $\begin{array}{c}\text { Amostra de 224 gestores } \\
\text { portugueses } \\
\text { (Rego et al., 2016) }\end{array}$ & $\begin{array}{c}\text { Amostra de 249 gestores } \\
\text { brasileiros } \\
\text { (Rego et al., 2016) }\end{array}$ \\
\hline Adaptabilidade & 3,92 & 3,86 & 4,05 \\
Valia dos mais velhos & 4,48 & 5,23 & 4,86 \\
Conscienciosidade e lealdade & 5,31 & 5,25 & 5,47 \\
Capital social e generosidade & 4,05 & 4,35 & 4,63 \\
Desempenho & 5,46 & 5,59 & 5,52 \\
\hline
\end{tabular}


que escolheria o mais novo é de $60 \%$. Globalmente, os dados sugerem que o pendor discriminatório dos estudantes é superior ao dos gestores (mais velhos). Não é possível saber se esta propensão provém das diferenças etárias, das ocupações dos participantes neste estudo e nos estudos de Rego et al., ou de ambas as razões. Como antes foi referido, a literatura (e.g., Cappelli \& Novelli, 2010; Kite et al., 2005) sugere que observadores mais jovens, comparativamente com os mais velhos, tendem a ser mais discriminatórios perante trabalhadores mais velhos. Mas é também possível que a experiência dos gestores lhes permita ser mais realistas a propósito do modo como interpretam a valia dos trabalhadores mais velhos.

Em quinto lugar, os nossos dados sugerem que as atitudes dos estudantes perante os trabalhadores mais velhos explicam algumas decisões que tomam perante os cenários. O poder explicativo é mais forte no caso em que a única caraterística que distingue os dois trabalhadores descritos no cenário é a idade (cenário 1). Quando outros elementos descritivos, favoráveis aos mais velhos, são facultados aos participantes no estudo, o poder preditivo das atitudes é menor. Essa evidência corrobora a obtida por Rego et al. (2016) e é consistente com a literatura (Kite et al., 2005).

Em suma, o estudo reforça a literatura anterior (Bendick \& Nunes, 2012; Daniel \& Heywood, 2007; Gordon \& Arvey, 2004; Phillips \& Siu, 2012; Posthuma et al., 2012), sugerindo que os mais velhos tendem a ser discriminados no contexto organizacional, mesmo quando lhes são reconhecidas qualidades importantes para a vida organizacional. Também é consistente com os dados de Rego et al. (2016) obtidos com gestores. $\mathrm{O}$ fato de estarmos perante uma amostra de estudantes universitários sugere que a tendência está porventura presente em diversos estratos populacionais, que se forma em idade precoce e que poderá persistir ao longo da vida profissional.

\section{Limitações e Estudos Futuros}

Este estudo comporta várias limitações, e pesquisas futuras poderão ser levadas a cabo para lidar com as mesmas. Primeira: os índices de consistência interna de duas dimensões ficam aquém do desejável. Estudos futuros poderão introduzir ou modificar itens de modo a melhorar as capacidades psicométricas da medida e garantir a sua aplicabilidade a diferentes tipos de respondentes/observadores. Segunda: apesar das cinco dimensões cobrirem um largo espectro de atitudes face aos trabalhadores mais velhos, estudos futuros deverão incluir atitudes adicionais (e.g., capacidades intelectuais e éticas). Terceira: algumas atitudes são medidas apenas através de itens com sentido semântico negativo. Estudos futuros deverão incluir itens formulados no sentido positivo. Quarta: os cenários apresentados para medição das variáveis dependentes cobrem um leque reduzido de possíveis decisões envolvendo trabalhadores mais velhos. Estudos futuros deverão incluir cenários distintos e mais complexos. Estudos futuros deverão, sobretudo, testar o poder preditivo das atitudes perante situações reais (em vez de hipotéticas), pois as decisões perante cenários não se traduzem, necessariamente, em decisões do mesmo teor perante situações reais. Embora as intenções sejam preditores dos comportamentos (Trafimow, 2004), são distintas dos mesmos.

Finalmente: a presente investigação focou-se no estudo das atitudes dos mais jovens relativamente aos trabalhadores mais velhos. Estudos futuros deverão estudar as atitudes dos trabalhadores mais velhos relativamente aos mais novos e testar o grau em que as mesmas explicam as relações intergeracionais no seio das organizações. São também recomendáveis estudos longitudinais que estudem as dinâmicas, ao longo do tempo, de como as atitudes dos membros de uma geração se repercutem nas atitudes dos membros da outra geração.

\section{Considerações Finais}

Apesar dessas limitações, o nosso estudo ajuda a compreender que (a) a diversidade de atitudes acerca dos trabalhadores mais velhos pode ser encontrada não apenas entre gestores, mas também em estudantes universitários, (b) essas atitudes ajudam a explicar ações de discriminação dos trabalhadores mais jovens, sobretudo quando a idade é a única variável que diferencia os trabalhadores sob consideração. $\mathrm{O}$ fato de essa evidência ter sido obtida com estudantes universitários, futuros profissionais e gestores, sugere a necessidade de o tema ser inserido nos programas universitários, designadamente através de conteúdos pedagógicos baseados na "evidência" (Pfeffer \& Sutton, 2007; Rynes, Giluk, \& Brown, 2007). A relevância do tema é mais que acadêmica.

\section{Referências}

American Psychological Association. (2002). Ethical principles of psychologists and code of conduct. Washington: American Psychological Association.

Ashbaugh, D. L., \& Fay, C. H. (1987). The threshold for aging in the workplace. Research on Aging, 9(3), 417-427. doi: $10.1177 / 0164027587093005$

Backes-Gellner, U., \& Veen, S. (2013). Positive effects of ageing and age diversity in innovative companies - large-scale empirical evidence on company productivity. Human Resource Management Journal, 23(3), 279-295. doi: 10.1111/17488583.12011

Backes-Gellner, U., Schneider, M. R., \& Veen, S. (2011). Effect of workforce age on quantitative and qualitative organizational performance: Conceptual framework and case study evidence. Organization Studies, 32(8), 1103-1121. doi: 10.1177/0170840611416746

Bendick, Jr., M., \& Nunes, A. P. (2012). Developing the research basis for controlling bias in hiring. Journal of Social Issues, 68(2), 238-262. doi: 10.1111/j.1540-4560.2012.01747.x

Billett, S., Dymock, D., Johnson, G., \& Martin, G. (2011). Overcoming the paradox of employers' views about older workers. International Journal of Human Resource Management, 22(6), 1248-1261. doi:10.1080/09585192.201 1.559097 
Byrne, B. M. (1998). Structural Equation Modelling with lisrel, prelis and simplis: Basic concept, application and programming. Mahwah, NJ: Lawrence Erlbaum

Capowski, G. (1994). Ageism: The new diversity issue. Management Review, 83(10), 10-15.

Cappelli, P., \& Novelli, B. (2010). Managing the older workers: How to prepare for the new organizational order. Boston, MA: Harvard Business Press.

Chiu, W.C.K., Chan, A. W., Snape, E., \& Redman, T. (2001). Age stereotypes and discriminatory attitudes towards older workers: An East-West comparison. Human Relations, 54(5), 629-661. doi: 10.1177/0018726701545004

Comissão Europeia. (2011). Active ageing and solidarity between generations: A statistical portrait of the European Union 2012. Luxembourg: Publications Office of the European Union. Retrieved from http://ec.europa.eu/ eurostat/documents/3217494/5740649/KS-EP-11-001-EN. PDF/1f0b25f8-3c86-4f40-9376-c737b54c5fcf, on February 24, 2014.

Cuddy, A. J. C., Norton, M. I., \& Fiske, S. T. (2005). This old stereotype: The pervasiveness and persistence of the elderly stereotype. Journal of Social Issues, 61(2), 267-285. doi: 10.1111/j.1540-4560.2005.00405.x

Daniel, K., \& Heywood, J. S. (2007). The determinants of hiring older workers: UK evidence. Labour Economics, 14(1), 35-51. doi: 10.1016/j.labeco.2005.05.009

DeLong, D. W. (2004). Lost knowledge: Confronting the threat of an aging workforce. Oxford: Oxford University Press.

Ekamper, P. (2010). Research note: Qualitative scenario study of the European Labour Force. Brussel: European Commission Directorate-General Employment.

Finkelstein, L. M., Burke, M. J., \& Raju, N. S. (1995). Age discrimination in simulated employment contexts: An integrative analysis. Journal of Applied Psychology, 80(6), 652-663. doi: 10.1037/0021-9010.80.6.652

Finkelstein, L. M., Ryan, K. M., \& King, E. B. (2013). What do the young (old) people think of me? Content and accuracy of age-based metastereotypes. European Journal of Work and Organizational Psychology, 22(6), 633-657. doi: 10.1080/1359432X.2012.673279

Gendron, B. (2011). Older workers and active ageing in France: The changing early retirement and company approach. International Journal of Human Resource Management, 22(6), 1221-1231. doi: 10.1080/09585192.2011.559095

Gordon, R. A., \& Arvey, R. D. (2004). Age bias in laboratory and field settings: A meta-analytic investigation. Journal of Applied Psychology, 34(3), 468-492. doi: 10.1111/j.1559-1816.2004. tb02557.x

Gringart, E., Helmes, E., \& Speelman, C. P. (2005). Exploring attitudes toward older workers among Australian employers. Journal of Aging \& Social Policy, 17(3), 85-103. doi: 10.1300/ J031v17n03_05

Hassell, B. L., \& Perrewe, P. L. (1995). An examination of beliefs about older workers: Do stereotypes still exist?. Journal of Organizational Behavior, 16(5), 457-468. doi: 10.1002/ job.4030160506

Kadefors, R., \& Hanse, J. J. (2012). Employers' attitudes toward older workers and obstacles and opportunities for the older unemployed to reenter working life. Nordic Journal of Working Life Studies, 2(3), 29-47. doi: 10.19154/njwls.v2i3.2362
Kanfer, R., \& Ackerman, P. L. (2004). Aging, adult development and work motivation. Academy of Management Review, 29(3), 440-458.

Kite, M. E., Stockdale, G. D., Whitley, Jr., B. E., \& Johnson, B. T. (2005). Attitudes toward younger and older adults: An updated meta-analytic review. Journal of Social Issues, 61(2), 241-266. doi: 10.1111/j.1540-4560.2005.00404.x

Larkey, L. B., \& Markman, A. B. (2005). Processes of similarity judgment. Cognitive Science, 29, 1061-1076. doi: 10.1207/ s15516709 cog0000_30

Leibold, M., \& Voelpel, S. C. (2006). Managing the aging workforce: Challenges and Solutions. New York: Wiley.

Liviatan, I., Trope, Y., \& Liberman, N. (2008). Interpersonal similarity as a social distance dimension: Implications for perception of others' actions. Journal of Experimental Social Psychology, 44, 1256-1269. doi:10.1016/j.jesp.2008.04.007

Lyon, P., \& Pollard, D. (1997). Perceptions of the older employee: Is anything really changing?. Personnel Review, 26(4), 245-257. doi: $10.1108 / 00483489710172051$

Masuch, M. (1985). Vicious circles in organizations. Administrative Science Quarterly, 30(1), 14-33.

Maurer, T. D., Barbeite, F. G., Weiss, E. M., \& Lippstreu, M. (2008). New measures of stereotypical beliefs about older workers' ability and desire for development. Journal of Managerial Psychology, 23(4), 395-418. doi: 10.1108/02683940810869024

Midtsundstad, T. I. (2011). Inclusive workplaces and older employees: An analysis of companies' investment in retaining senior workers. International Journal of Human Resource Management, 22(6), 1277-1293. doi: 10.1080/09585192.2011.559099

Munnell, A. H., Sass, S. A., \& Soto, M. (2006). Employer attitudes toward older workers: Survey results. Boston: Center for Retirement Research, Boston College.

Nelson, T. D. (2005). Ageism: Prejudice against our feared future self. Journal of Social Issues, 61(2), 207-221. doi: 10.1111/j.1540-4560.2005.00402.x

OECD. (2006). Live longer, work longer. Paris: OECD Publishing.

OECD. (2012). Pensions Outlook 2012. Paris: OECD Publishing. DOI: $10.1787 / 9789264169401-e n$.

Pfeffer, J., \& Sutton, R. (2007). Suppose we took evidencebased management seriously: Implications for reading and writing management. Academy of Management Learning and Education, 6, 153-155. doi: 10.5465/AMLE.2007.24401715

Phillips, D. R., \& Siu, O. L. (2012). Global Aging and Aging Workers. In J. W. Hedge \& W. C. Borman (Eds.), The Oxford handbook of work and aging (pp. 11-32). New York: Oxford University Press.

Posthuma, R. A., \& Campion, M. A. (2009). Age stereotypes in the workplace: Common stereotypes, moderators, and future research directions. Journal of Management, 35, 158-188. doi: $10.1177 / 0149206308318617$

Posthuma, R. A., Wagstraff, M. F., \& Campion, M. A. (2012). Age stereotypes and workplace age discrimination - A framework for future research. In J. W. Hedge \& W. C. Borman (Eds.), The Oxford Handbook of Work and Aging (pp. 298-312). New York: Oxford University Press.

Price, J. L., \& Mueller, C. W. (1986). Handbook of organizational measurement. Marshfield, MA: Pitman. 
Rego, A., Vitória, A., Tupinambá, A., Cunha, M. P., \& Leal, S. (2016). Development and validation of an instrument for measuring managers' attitudes toward older workers. International Journal of Human Resource Management. DOI: 10.1080/09585192.2015.1128462.

Roberson, L., \& Kulik, C. (2007). Stereotype threat at work. Academy of Management Perspectives, 21(2), 24 - 40. doi: 10.5465/AMP.2007.25356510

Rynes, S., Giluk, T. L., \& Brown, K.G. (2007). The very separate worlds of academic and practitioner periodicals in human resource management: Implications for evidence-based management. Academy of Management Journal, 50, 987- 1008. doi: 10.5465/AMJ.2007.27151939

Schalk, R., van Veldhoven, M., de Lange, A. H., de Witte, H., Kraus, K., Stamov-Roßnagel, C., ... Zacher, H. (2010). Moving European research on work and ageing forward: Overview and agenda. European Journal of Work and Organizational Psychology, 19(1), 76-101. doi: 10.1080/13594320802674629

Skirbekk, V., Loichinger, E., \& Barakat, B. F. (2012). The aging of the workforce in European countries - Demographic trends, retirement projections, and retirement policies. In J. W. Hedge \& W. C. Borman (Eds.), The Oxford handbook of work and aging (pp. 60-79). New York: Oxford University Press.
Streb, C. K., Voelpel, S. C., \& Leibold, M. (2008). Managing the aging workforce: Status quo and implications for the advancement of theory and practice. European Management Journal, 26(1), 1-10. doi: 10.1016/j.emj.2007.08.004

Taylor, P., \& Walker, A. (1998). Employers and older workers: Attitudes and employment practices. Aging and Society, 18(6), 641-658.

Tikkanen, T. (2011). From managing a problem to capitalizing on talent and experience of older workers. International Journal of Human Resource Management, 22(6) 1217-1220. doi: 10.1080/09585192.2011.559093

Trafimow, D. (2004). Attitude measurement. In C. Spielberger (Ed.), Encyclopedia of Applied Psychology (pp. 233-244). New York: Elsevier Academic Press.

Villosio, C. (2008). Working conditions of an ageing workforce. Luxembourg: Office for Official Publications of the European Communities.

von Bonsdorff, M. E. (2011). Age-related differences in reward preferences. International Journal of Human Resource Management, 22(6), 1262-1276. doi: $10.1080 / 09585192.2011 .559098$

Warr, P. (1994). Age and employment. In H. C. Triandis, M. D. Dunnette \& L. M. Hough (Eds.), Handbook of industrial and organizational psychology (Vol. 4, pp. 485-550). Palo Alto, CA: Consulting Psychologists Press

Recebido em 29.07.2014

Primeira decisão editorial em 03.11.2014

Versão final em 12.12.2014

Aceito em 12.12.2014 